\title{
ARTICLES
}

\section{Calculated structural-dependent optical properties of alloys TiNi, TiPd, and TiPt}

\author{
Xindong Wang \\ Metals and Ceramics Division, Oak Ridge National Laboratory, Oak Ridge, Tennessee 37831-6114 \\ Y. Y. Ye \\ Center of Analysis and Testing, Wuhan University, Wuhan, China \\ C. T. Chan \\ Department of Physics, Hong Kong University of Science and Technology, Hong Kong, China \\ K. M. Ho and B. N. Harmon \\ Department of Physics and Astronomy, Iowa State University, Ames, Iowa 50014 \\ (Received 23 October 1997; revised manuscript received 5 February 1998)
}

\begin{abstract}
Electronic structures and optical properties of martensitic alloys TiNi, TiPd, and TiPt in both the lowtemperature $B 19\left(B 19^{\prime}\right.$ in the case of $\left.\mathrm{TiNi}\right)$ and the high-temperature $B 2$ phases are systematically calculated using the flat-principles local density functional approach. Our results on TiNi $B 2$ and $B 19^{\prime}$ phases agree well with the existing experimental data where drastic changes of optical conductivity in the infrared region from $B 2$ to $B 19^{\prime}$ phase were observed. Our results show that optical matrix elements are important in understanding the optical properties of these alloys, especially in obtaining the correct peak intensities. Because of the different crystalline symmetry between the low-temperature $B 19\left(B 19^{\prime}\right.$ in the case of TiNi) phase and the high-temperature $B 2$ phase, different polarization dependence in the optical conductivity of the two phases is expected. We present the polarization-dependent optical conductivities in these martensitic alloys. [S0163-1829(98)02430-8]
\end{abstract}

\section{INTRODUCTION}

Martensitic alloys have attracted considerable research attention due to their many fascinating mechanical properties such as pseudoelasticity and the shape-memory effect. It is generally accepted that a martensitic transformation, a type of diffusionless structural phase transformation involving changes in the shape of the unit cell as well as atomic position displacements within the unit cell, is responsible for many of the exotic mechanical properties exhibited. Among these alloys, the Ti family of binary intermetallics represents one of the most intensively studied classes of materials both experimentally and theoretically.

Both experimental evidence ${ }^{1}$ and theoretical investigations ${ }^{2}$ have suggested that the martensitic transformation is of electronic nature for Ti-based shape memory alloys. This point of view has further stimulated many investigations exploring the differences in electronic structures of different structural phases involved in the transformation, namely, the low-temperature $B 19$ (or $B 19^{\prime}$ ) phase and the high-temperature $B 2$ phase. Since optical conductivity in the infrared region is usually sensitive to the energy band structure near the Fermi level, the study of it will provide useful information on the electronic structure differences between different structural phases.

Measurement of optical conductivity in the TiNi alloy was reported by Sasovskaya ${ }^{3}$ in the energy range of $0.062-$
$4.96 \mathrm{eV}$ for both the $B 2$ and $B 19^{\prime}$ phases. While the spectra above $1.5 \mathrm{eV}$ show a close resemblance between the two phases, they show a pronounced difference below $1.5 \mathrm{eV}$. Since this drastic difference below $1.5 \mathrm{eV}$ was not reproduced by a calculation ${ }^{4}$ in which a constant matrix element was assumed, Sasovskaya concluded that the theoretical band structure was to be questioned. However, these previous calculations ${ }^{4,5}$ did not include the optical matrix elements which are important for obtaining accurate results, particularly the peak intensities.

In this paper, we present theoretical results of the interband contributions to the optical conductivity based on local density functional energy bands. We have included the calculated optical matrix elements to obtain the optical conductivity spectra. We found that they are important in obtaining better agreement with the experimental results for the TiNi alloy. More specifically, we reproduce the observed large difference below $1.5 \mathrm{eV}$ in the optical conductivity spectra for the two phases of the TiNi alloy.

Because of the different crystalline symmetry between the low-temperature $B 19\left(B 19^{\prime}\right.$ in the case of TiNi) phase and the high-temperature $B 2$ phase, different polarization dependence in the optical conductivity of the two phases is expected. We present the polarization-dependent optical conductivities in these martensitic alloys. We hope to motivate future experimental measurements on single crystals which will verify our theoretical predictions. 


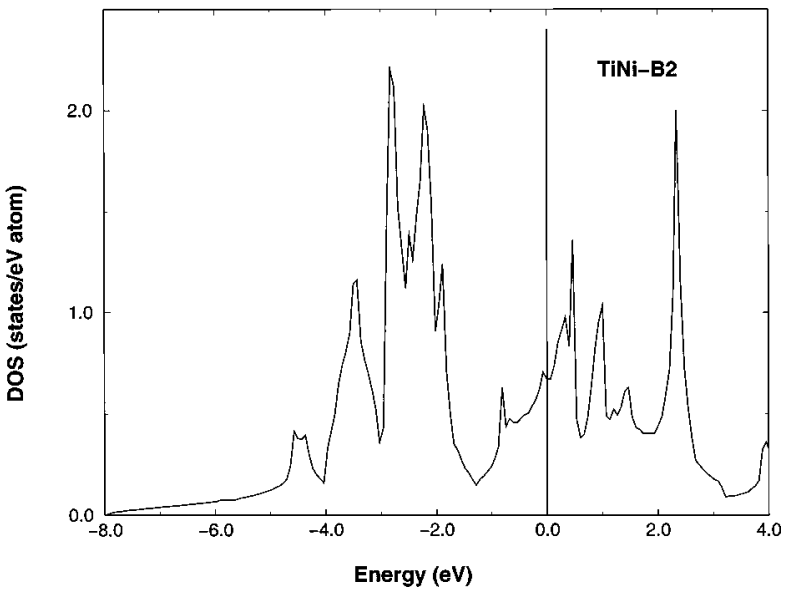

FIG. 1. Total density of states of TiNi in the $B 2$ phase.

\section{METHOD OF CALCULATION}

The energy band calculations for all three alloys TiNi, TiPd, and TiPt, were carried out using the self-consistent tight-binding linear muffin-tin orbital method in the atomic sphere approximation with combined correction (TB-LMTO-ASA). ${ }^{6}$ A Hedin-Lundqvist ${ }^{7}$ form for the local exchange-correlation functional is used. Once we obtained the self-consistent potential and the band structure, the interband contribution to the complex optical conductivity at energy $\omega$ was calculated via

$$
\begin{aligned}
\sigma_{\alpha}(\omega)= & \frac{i e^{2}}{\hbar m^{2} \Omega} \sum_{n, l} f\left(\omega_{l}\right)\left[1-f\left(\omega_{n}\right)\right] \frac{\left|\left\langle n\left|p_{\alpha}\right| l\right\rangle\right|^{2}}{\omega_{n l}} \\
& \times\left(\frac{1}{\omega-\omega_{n l}+i \epsilon}+\frac{1}{\omega+\omega_{n l}+i \epsilon}\right)
\end{aligned}
$$

where $\epsilon$ is a positive infinitesimal, $p_{\alpha}=\mathbf{p} \cdot \mathbf{n}_{\alpha}$ in which $\mathbf{n}_{\alpha}$ is the polarization direction of the photons, $\Omega$ is the unit cell volume, and $f(\omega)$ is the Fermi-Dirac occupation function.

Since all of the structures under study have BCC-like atomic packing, no empty sphere was found necessary for the ASA approximation. In obtaining the self-consistent potential, both $l_{\max }=2(d)$ and $l_{\max }=3(f)$ have been used and our results remain the same.

However, it is found that in optical calculations, including the $f$ component in the matrix element calculations makes a significant difference in the intensities of the spectra calculated. This suggests that $d-f$ transitions in $d$-electron systems cannot be neglected. A detailed discussion of the role of the $d-f$ transition in optics can be found in Ref. 8. Since we performed matrix element calculations within the atomic spheres, i.e., no combined correction for matrix elements is included, a definitive understanding to this $l$ sensitivity has to await a more accurate full potential calculation.

The Brillouin zone (BZ) integration is carried out using the linear tetrahedron method. ${ }^{9,10}$ For the self-consistent energy band calculations, a $10 \times 10 \times 10$ division of the $\mathrm{BZ}$ is used, while for optical conductivity calculations, a $12 \times 12$ $\times 12$ division of the BZ is used. Group theory is utilized to generate the number of irreducible $k$ points. In the optical calculation, whenever the Fermi surface cuts through a tetrahedron, further division of the tetrahedron is performed so

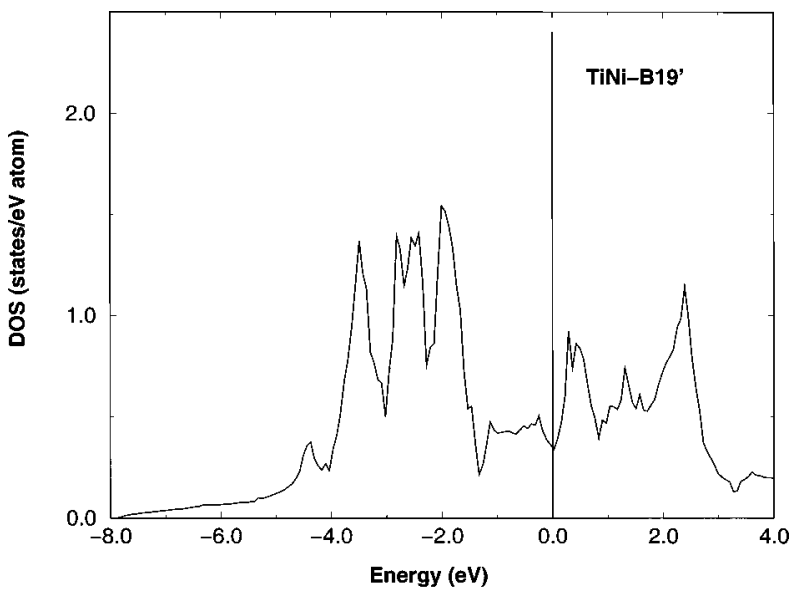

FIG. 2. Total density of states of TiNi in the $B 19^{\prime}$ phase.

that for the remaining smaller tetrahedra, the occupation factor $f\left(\omega_{l}\right)\left[1-f\left(\omega_{n}\right)\right]=1$ is satisfied. We have found that this further division is important for the convergence of our results in the low-energy region. To check the convergence of the BZ integration, we have compared results for the $B 2$ structure from three different meshes, obtained by a $10 \times 10$ $\times 10$ division, a $12 \times 12 \times 12$ division, and a $14 \times 14 \times 14$ division of the BZ, and all three agree well with each other
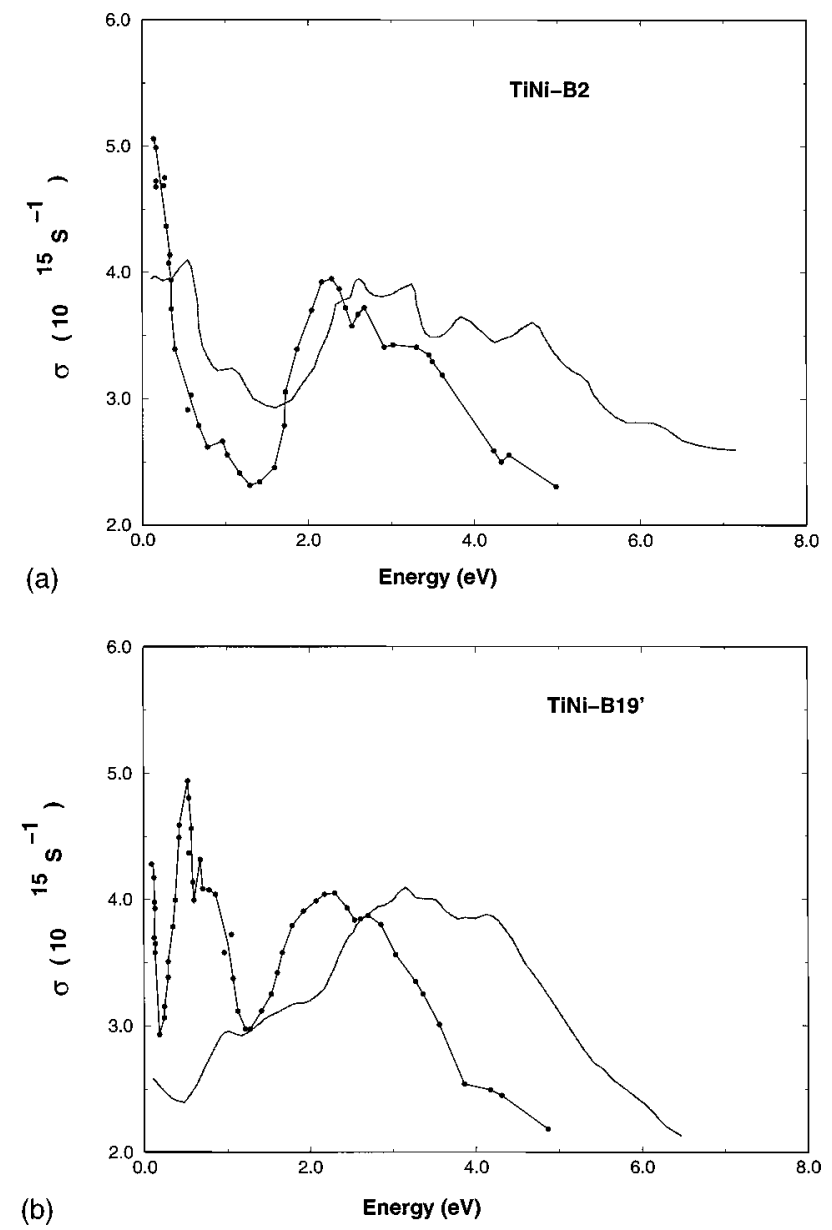

FIG. 3. Experimental data (Ref. 3) (solid line with data points) and theoretical results (Ref. 5) (grey line) reproduced for (a) TiNi $B 2$ phase and (b) TiNi $B 19^{\prime}$ phase. 


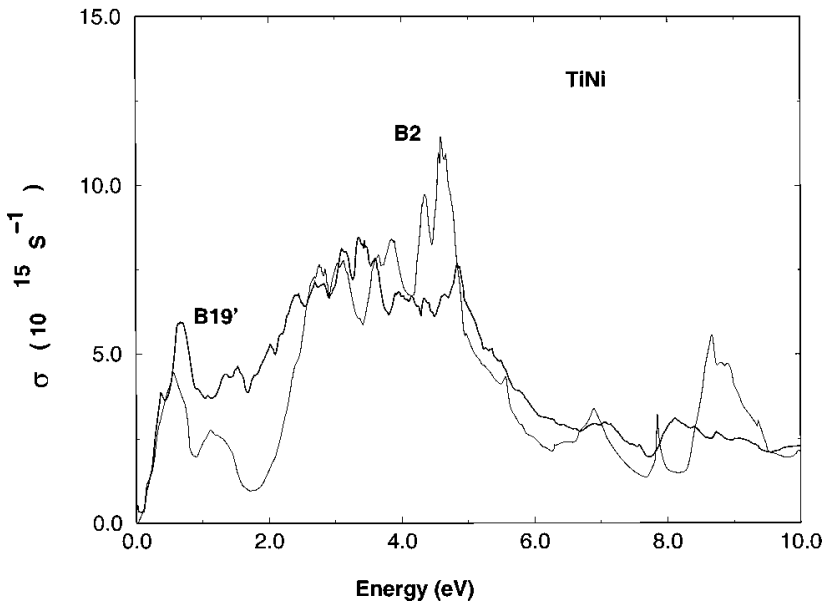

FIG. 4. Calculated optical conductivities for TiNi $B 2$ and $B 19^{\prime}$ phases.

with the latter two results almost identical. Since the $B 19$ and $B 19^{\prime}$ structure have a smaller BZ zone, we believe that a $12 \times 12 \times 12$ division is sufficient for our calculations. We want to point out an important issue in calculating the polarization dependence of the optical conductivity: a full employment of the crystal symmetry in the reduction of $k$ points or tetrahedra can often lead to spurious polarization dependence. This is because equivalent $k$ points related by symmetry do not always have the same matrix elements for a specific polarization. For example, $\mathbf{k}_{1}=\left(k_{x}, k_{y}, k_{z}\right)$ and $\mathbf{k}_{2}=\left(k_{y}, k_{x}, k_{z}\right)$ are two equivalent $k$ points for $B 2$ structure, while in general $\left\langle\psi_{i}\left(\mathbf{k}_{1}\right)\left|p_{x}\right| \psi_{j}\left(\mathbf{k}_{1}\right)\right\rangle \neq\left\langle\psi_{i}\left(\mathbf{k}_{2}\right)\left|p_{x}\right| \psi_{j}\left(\mathbf{k}_{2}\right)\right\rangle$. In our polarization dependence calculations, we only used symmetry elements that also keep the polarization specific matrix elements invariant.

In all of our calculations, the structural parameters are those given by Ye, Chan, and $\mathrm{Ho}^{11}$ which were fully optimized using first-principles pseudopotential total energy and atomic force calculations.

\section{RESULTS AND DISCUSSIONS}

Among the three alloys under study the optical properties of TiNi and its structural dependence have been best characterized experimentally ${ }^{3}$ and most intensively studied

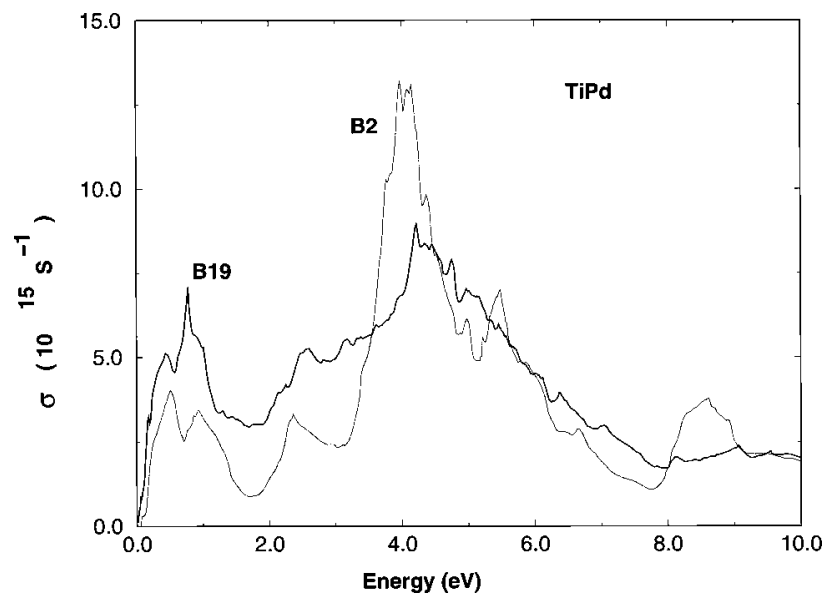

FIG. 5. Calculated optical conductivities for TiPd $B 2$ and $B 19$.

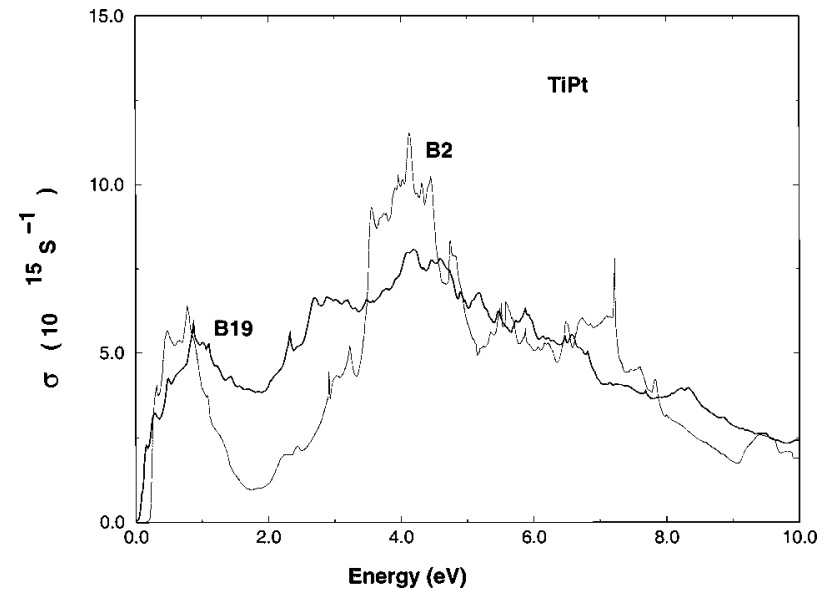

FIG. 6. Calculated optical conductivities for TiPt $B 2$ and $B 19$.

theoretically. ${ }^{4,5,12}$ Section III A will be focused on the optical properties of TiNi, followed by results for TiPd and TiPt in Sec. III B. Section III C will show the photon polarization dependence of the optical conductivities of the $B 19^{\prime}$ phase of TiNi, and the $B 19$ phases of TiPd and TiPt.

\section{A. Optical conductivities of TiNi $B 2$ and $B 19^{\prime}$ phases}

The total density of states (DOS) of TiNi $B 2$ and $B 19^{\prime}$ phases are shown in Figs. 1 and 2, respectively. While our results on the electronic structure of the TiNi $B 2$ phase agree well with the published results, ${ }^{5,12}$ we have found considerable difference between our $B 19^{\prime}$ results and those of Ref. 5: our total DOS (shown in Fig. 2) clearly shows that the Fermi level for the TiNi $B 19^{\prime}$ phase falls in the dip of a pseudogap, which is not observed in Ref. 5. Since for all the systems studied, our TB-LMTO-ASA code gives an almost identical DOS and very similar band structure as compared to the pseudopotential calculations, ${ }^{11}$ provided that the same structural parameters are used, we believe that the different structural parameters used in the present work and in Ref. 5 are responsible for the differences observed in the TiNi $B 19^{\prime}$ phase.

The experimental data of optical conductivities for TiNi $B 2$ and $B 19^{\prime}$ phases $^{3}$ along with the theoretical results of

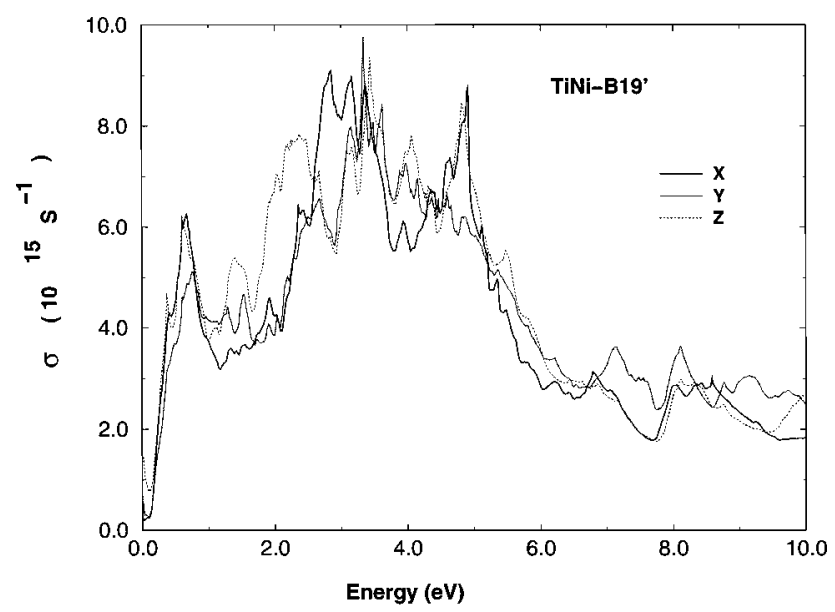

FIG. 7. Polarization resolved optical conductivities for TiNi $B 19^{\prime}$ phase. For an explanation of the three directions, see text. 


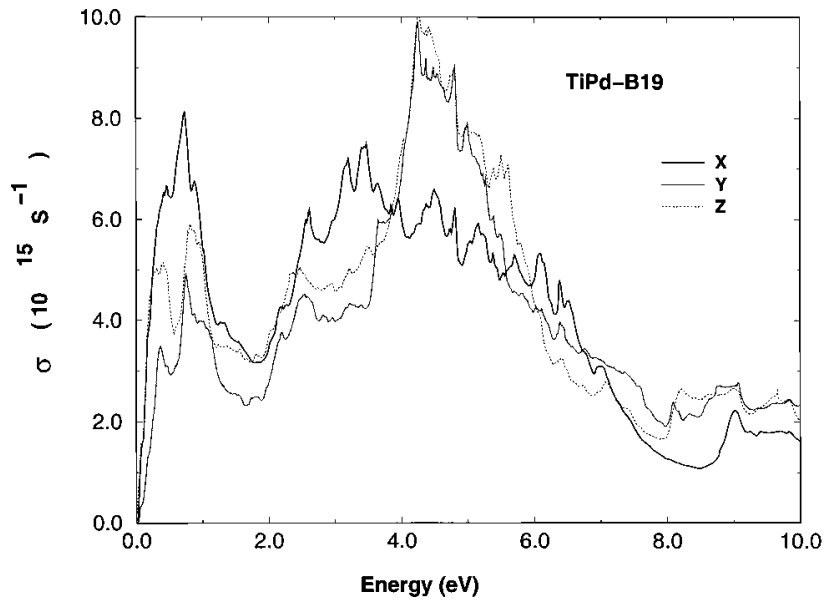

FIG. 8. Polarization resolved optical conductivities for TiPd B19 phase. For an explanation of the three directions, see text.

Ref. 5 are reproduced in Fig. 3. It is noted that the result of Ref. 5, which assumes constant matrix elements, could not reproduce the intensive absorption peak observed in the TiNi $B 19^{\prime}$ phase in the region of 0.5 to $1 \mathrm{eV}$. In contrast, our calculated results shown in Fig. 4 are in much better agreement with experiment. ${ }^{3}$ Specifically, our results clearly show a much stronger optical absorption in the TiNi $B 19^{\prime}$ phase than that in the $B 2$ phase below $2 \mathrm{eV}$ and an intensive peak in the region of $0.5 \mathrm{eV}$ to $1 \mathrm{eV}$ for the $B 19^{\prime}$ phase. We have found that the inclusion of matrix elements is very important in getting the correct peak intensities reported in this work.

In closing this subsection, we note that the results reported here do not include the intraband contributions, which usually can be well described by the Drude form $\sigma(0) /[1$ $\left.+(\omega \tau)^{2}\right]$, in which the dc conductivity $\sigma(0)$ is proportional to the relaxation time $\tau$ and $\hbar / \tau$ is the width of the Drude term. We did not calculate the plasma frequency in this work. Since in the long wavelength limit, the intraband contribution dominates, we have estimated from experimental data shown in Fig. 3 that the inverse relaxation time $\hbar / \tau$ is roughly 0.2 and $0.1 \mathrm{eV}$ for TiNi $B 2$ and $B 19^{\prime}$ phases, respectively. Therefore the intraband absorption is not expected to contribute much to the optical conductivity above $0.5 \mathrm{eV}$ in these systems.

\section{B. Structural-dependent optical conductivities of TiPd and TiPt}

Common to all three alloys studied in this work, the change of electronic structures from $B 2$ phase to $B 19$ phase is the opening up of a pseudogap so that the Fermi level falls into the dip of the pseudogap. ${ }^{11}$ Because of this change in the electronic structure, we expect changes in the optical conductivities in the low-energy region (less than $2 \mathrm{eV}$ ). Figures 5 and 6 show the calculated optical conductivities of TiPd and TiPt, respectively. For both TiPd and TiPt, stronger optical absorptions below $2 \mathrm{eV}$ take place in the $B 19$ phase than in the $B 2$ phase, a trend similar to that in TiNi. However, the peak intensities around $1 \mathrm{eV}$ for TiPt, unlike those for TiNi and TiPd, do not show much difference between $B 2$ and $B 19$ phases. This suggests that the analysis of the optical conductivity data requires very detailed theoretical calculations.

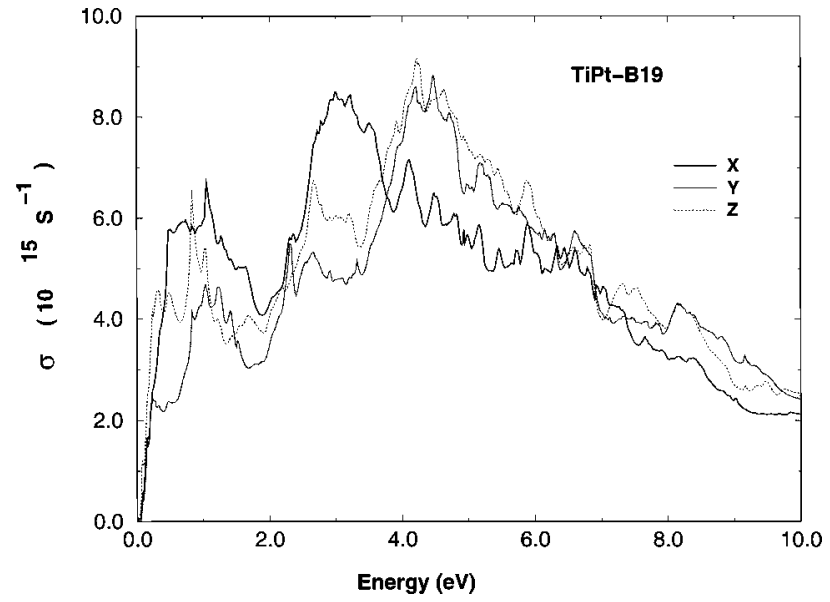

FIG. 9. Polarization resolved optical conductivities for TiPt B19 phase. For an explanation of the three directions, see text.

\section{Photon polarization dependence of the optical conductivities}

As pointed out in the Introduction, the structural transformation from $B 2$ to $B 19$ ( $B 19^{\prime}$ in the TiNi case) involves a reduction of symmetry. While for the $B 2$ phase with cubic symmetry, no photon polarization dependence of the optical conductivity is expected; this is not true for $B 19$ or $B 19^{\prime}$ phases. Figures 7, 8, and 9 show the calculated conductivities for polarizations along the $x, y$, and the $z$ directions. Some clarification for these polarization directions is in order. For the TiNi $B 19^{\prime}$ phase, only $x$ and $z$ directions are along the crystalline axis and the $z$ direction coincides with the monoclinic $c$ direction and $x$ is along the crystal axes with the shortest length of the unit vector. For the $B 19$ phase, we arrange the lattice parameter in the order of $a<c<b$ and $x, y, z$ directions in Figs. 8 and 9 are along the $a, b, c$ unit axes respectively. The optical conductivities shown in Figs. 4,5 , and 6 for $B 19^{\prime}$ or $B 19$ phases are the ones averaged over the three polarizations. In order to ensure that our calculated polarization dependence is not spurious, we have made the same calculations for the $B 2$ structure compounds and no polarization dependence was found in all three alloys, as expected.

The measurement of polarization-dependent optical conductivity requires good single crystal samples and more sophisticated techniques. More work is needed for the $B 19$ or $B 19^{\prime}$ phase. When the transformation takes place in a single crystal in the $B 2$ phase, domains of the $B 19$ or $B 19^{\prime}$ phases will form which will average out the polarization dependence. A possible way to promote growth of the domains with a selected crystalline alignment is to strain the crystal. We hope that the present work will stimulate future experimental inquiry along this line.

Finally we want to point out that the apparent nonzero interband optical conductivities at the lowest-energy mesh (0.001 Ryd) shown in some of our results are not due to integration errors. In general this happens when (a) there are two bands close to each other (energy difference 0.001 Ryd) and both of them cross the Fermi surface in some finite region of the BZ, and (b) the interband optical matrix elements between these two bands are finite. A more detailed analysis of the interband contribution to the infrared optical properties can be found in Ref. 13 . 


\section{ACKNOWLEDGMENTS}

The authors thank Dr. T. C. Schulthess and Dr. G. S. Painter for their helpful comments on the manuscript. The research of X.W. was performed as a staff member at the Oak Ridge National Laboratory and was sponsored by the Division of Materials Sciences, Office of Basic Energy Sciences, U.S. Department of Energy under Contract No. DE-
AC05-96OR22464 with Lockheed Martin Energy Research Corporation. Y.Y.Y. is grateful to Ames Lab. (USDOE) for its hospitality, and was supported by the Chinese National Science Foundation. C.T.C. was supported by RGC Hong Kong through Grant No. DAG95/96-SC12 from HKUST. Ames Laboratory is operated for the U.S. Department of Energy by Iowa State University under Contract No. W-7405ENG-82.
${ }^{1}$ S. Shabalovskaya, A. Narmonev, O. Ivanova, and A. Dementjev, Phys. Rev. B 48, 13296 (1993); S. Shabalovskaya, in ShapeMemory Materials and Phenomena-Fundamental Aspects and Applications, edited by C. T. Liu, H. Kunsmann, K. Otsuka, and M. Wuttig, MRS Symposia Proceedings No. 246 (Materials Research Society, Pittsburgh, 1992), p. 247.

${ }^{2}$ G. L. Zhao and B. N. Harmon, Phys. Rev. B 48, 2031 (1993).

${ }^{3}$ I. I. Sasovskaya, Phys. Met. Metallogr. 69, 72 (1990).

${ }^{4}$ V. Ye. Yegorushkin and N. I. Fedyaynova, in Alloys of Rare Metals with Special Physical Properties (Nauka, Moscow, 1983), p. 24.

${ }^{5}$ S. E. Kul'kova, K. A. Beketov, V. E. Egorushkin, and O. N. Muryzhnikova, J. Phys. IV C8, 539 (1995).

${ }^{6}$ O. K. Andersen, O. Jepsen, and D. Gloetzel, in Highlights of
Condensed Matter Theory, edited by F. Bassani, F. Fumi, and M. P. Tosi (North-Holland, New York, 1985).

${ }^{7}$ L. Hedin and B. I. Lundqvist, J. Phys. C 4, 2064 (1971).

${ }^{8}$ Yu. A. Uspenski, E. G. Maksimov, S. N. Rashkeev, and I. I. Mazin, Z. Phys. B 53, 263 (1983).

${ }^{9}$ O. Jepsen and O. K. Andersen, Solid State Commun. 9, 1763 (1971).

${ }^{10}$ O. Jepsen and O. K. Andersen, Phys. Rev. B 29, 5965 (1984).

${ }^{11}$ Y. Y. Ye, C. T. Chan, and K. M. Ho, Phys. Rev. B 56, 3678 (1997).

${ }^{12}$ Joo Yull Rhee, B. N. Harmon, and D. W. Lynch, Phys. Rev. B 54, 17385 (1996), and references therein.

${ }^{13}$ S. N. Rashkeev, Yu. A. Uspenski, and I. I. Mazin, Sov. Phys. JETP 61, 1004 (1985). 